\section{A SCENE AND A PHOTO PROGRAMA CONECTAR IGUALDAD AND POPULAR EDUCATION}

RECIBIDO 14 | 07 | 2016

ACEPTADO $27|10| 2016$

\section{Una escena y una foto Programa Conectar Igualdad y educación popular}

\author{
Darío Martínez \\ dariogmartinez@gmail.com \\ http://orcid.org/0000-0003-2415-8761
}

Consejo Nacional de Investigaciones Científicas y Técnicas (CONICET)

Laboratorio de investigación en Comunicación, Medios,

Educación y Discurso (COMEDI)

Facultad de Periodismo y Comunicación Social

Universidad Nacional de La Plata | Argentina
Palabras clave

comunicación

educación

jóvenes

tecnologías

\section{Resumen}

La relación de la educación y las tic ha sido tensa, con celebraciones acríticas y con optimismos ficticios. El Programa Conectar Igualdad tampoco estuvo exento de estas tensiones y de operaciones discursivas que buscaron cuestionar su alcance. Esto se transformó en un punto de partida para comenzar a indagar en algunas de las experiencias más reconocidas de comunicación y educación popular y en los vínculos que estas establecieron con las tic disponibles en cada momento histórico. Con ello, se intenta trazar un horizonte de intervención que busque interpelar a los/as jóvenes para promover lecturas del mundo que desafíen a las hegemónicas.

\section{Abstract}

Keywords communication education

youth

technologies

The relationship of education and ict has been tense, with celebrations and fictitious uncritical optimism. The Equal Connect program was not exempt from these tensions and discursive operations that sought to question its scope. This became a starting point to begin to delve into some of the most recognized and popular communication experiences education and the links established with the available ict in each historical moment. With this, we draw a horizon of intervention seeking to interpellate young people to promote reading in the world that challenge hegemonic. 


\title{
Una escena y una foto Programa Conectar Igualdad y educación popular
}

\author{
Por Darío Martínez \\ No estoy entre los conquistados, \\ sino entre los derrotados a los que los vencedores temen. \\ El tiempo de los vencedores es siempre corto \\ y el de los derrotados es inconmensurablemente largo.
}

John Berger

\section{La escena}

El lunes 10 [de febrero de 2014] nació Amanda. Las fotos indican que tiene algo mío: el gorrito de recién nacida no le entraba en la cabeza.

En la habitación 222 del Hospital Español, había una pareja de jóvenes (mucho más que nosotros, lo eran sus rostros, sus cuerpos) que habían tenido una nena el viernes. Casualmente, la llamaron Simona como nuestra primera hija. Un par de horas después le dan el alta a la mamá que todavía estaba dolorida por la cesárea. Comienza la escena de los preparativos para irse. La mamá chancletea con gestos de molestia por la operación. El papá inicia la tarea de cargarse bolsos, bolsitos y cambiador. A ese cargamento se le sumó en su mano derecha una netbook del Plan Conectar lgualdad. 
Esa escena me dejó regulando. Una política pública inclusiva trasciende su ámbito de incidencia directa para irradiar a otras zonas de la cotidianeidad. (Era febrero y ni siquiera estaban las fechas de las mesas de exámenes). Se amplía el mundo de las experiencias de los destinatarios. En este caso, el umbral de sus horizontes de vida se ensancha.

\section{La foto}

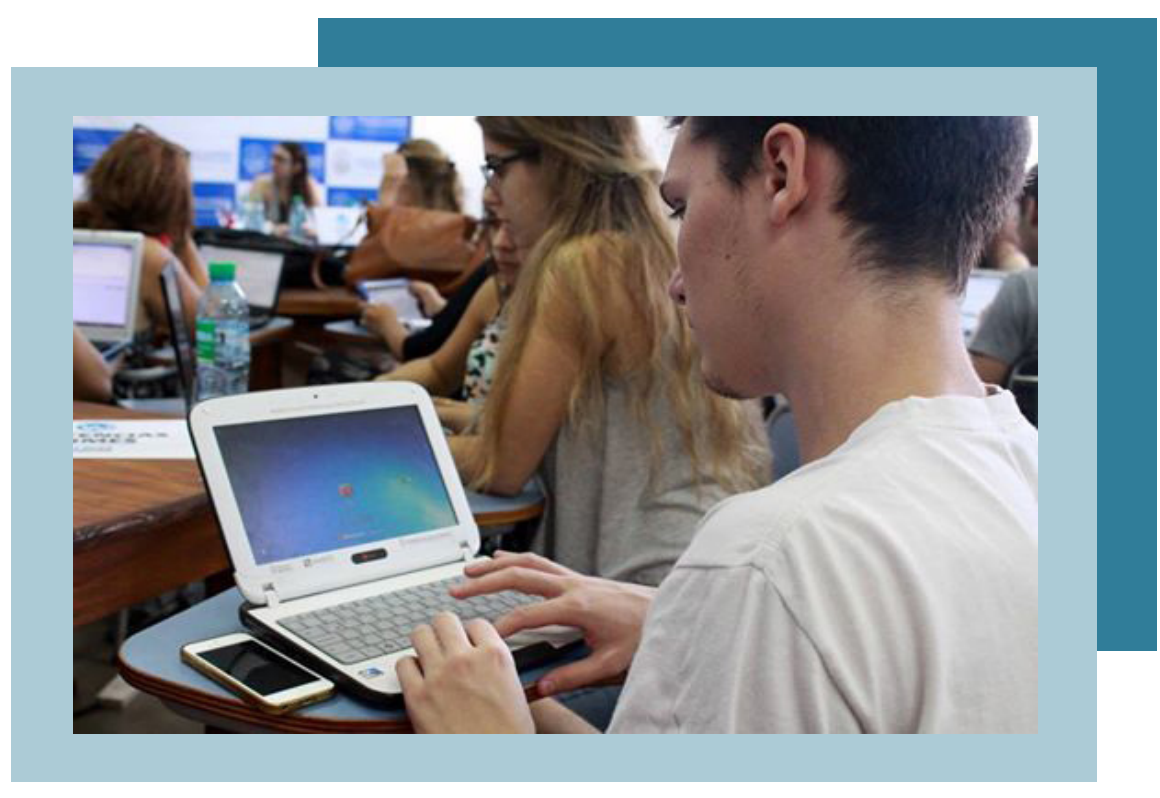

Esta foto se tomó en la Facultad de Periodismo y Comunicación Social de la Universidad Nacional de La Plata el 17 de marzo de 2016, durante el Taller de Edición «EDITATÓN WIKILESA a 40 años del Golpe». La propuesta fue convocada por la Facultad en conjunto con Wikimedia Argentina y con Cosecha Roja. Estudiantes de carreras de grado y de posgrado produjeron colaborativamente textos vinculados a la lucha por los derechos humanos y los juicios por los crímenes de lesa humanidad, y luego los subieron a la enciclopedia libre para enriquecer sus contenidos. 
La imagen muestra a un estudiante de grado participando del taller. Allí está con su computadora del Conectar Igualdad, con celular a su lado y con el índice de la mano derecha dirigiendo el cursor, en un ámbito universitario escribiendo contenidos acerca de los derechos humanos para aportar información a la enciclopedia Wikipedia.

\section{Los puntos de partida}

Deliberadamente, opté por comenzar a reflexionar sobre el Programa Conectar lgualdad (PCl) y los jóvenes a partir de acontecimientos desarrollados en un ámbito privado, muy íntimo, y otro en uno público. Ambos condensan un núcleo de problemáticas y prácticas juveniles, que resultan ineludibles para comprender las transformaciones culturales y las nuevas formas de organización política que estructurarán los antagonismos sociales en este siglo xxı. La escena y la foto tienen como protagonistas a jóvenes que enfrentan los contratiempos de la vida en común y realizan lecturas del mundo mediadas por las tecnologías de la información y la comunicación. Ahí es posible conjeturar horizontes políticos que permitan comprender los alcances del programa, los modos de apropiación, pero sobre todo las prácticas emancipatorias que puedan habilitar experiencias de toma de la palabra.

El PCl se creó mediante el Decreto 459/10 por parte del Estado nacional. Hasta la actualidad, se han entregado aproximadamente cinco millones de netbooks en todo el país. Entre sus principales fundamentos se menciona que se trata de una iniciativa destinada a revalorizar la escuela pública, mejorar los aprendizajes y reducir las brechas sociales y educativas a través de la provisión de netbooks a alumnos y docentes, la actualización de las formas de enseñanza, el fortalecimiento del rol docente y la producción de recursos y contenidos pedagógicos. Al mismo tiempo, explica:

La implementación del Programa Conectar Igualdad implica la definición de una política pública de carácter universal que garantiza, no solo el acceso a las nuevas tecnologías de la información y la comunicación, sino el derecho a una educación de calidad acorde a la realidad de nuestros jóvenes y al desarrollo de nuestro país en términos económicos, sociales, culturales y políticos (Ministerio de Educación, 2014: 14). 
El programa trascendió la mera entrega de netbooks; incluyó, además, estrategias de formación docente destinadas - en un primer momento- al manejo de los programas que traían las computadoras, a partir de cursos virtuales. También se realizaron propuestas que abarcaron a otros organismos del Estado, como es el caso del Instituto Nacional de Formación Docente (INFD), que lanzó una Especialización en Educación y TIC, una carrera con dos años de duración y con cuatrocientas horas de cursada mediante la plataforma virtual del programa. Esta propuesta comenzó en 2012 y tiene cinco mil egresados en todo el país (Artiguenave, 2015).

En la actualidad, durante la gestión del presidente Mauricio Macri se han producido miles de despidos de trabajadores y de trabajadoras de distintos organismos del Estado. El PCl no ha sido la excepción. Quienes primero se vieron afectados por estas medidas fueron los profesionales dedicados a la coordinación pedagógica del programa, haciéndose evidente hacia dónde quiere direccionar el programa la decisión política: en una suerte de distribución de dispositivos tecnológicos. Asimismo, son constantes las presiones de las corporaciones informáticas que anhelan obtener los contratos millonarios de un mercado inmenso y cautivo como es el de los destinatarios de la educación.

Hasta aquí el panorama, que dista de ser alentador. Sin embargo, en la escena y en la foto que se describieron se hallan elementos que intersectan las prácticas generadas por el PCl y las memorias de comunicación y educación popular. Quizás en esto radique una clave para tajear esta opaca y densa coyuntura.

\section{Las memorias de lo que podemos ser}

La relación de la educación y las Tıc ha sido tensa, con celebraciones acríticas y con optimismos ficticios. Con matices, se han realizado movimientos pendulares entre una posición y otra, según los énfasis que cada agente quiso asignarle a la práctica o al discurso que hiciera evidente esta relación. Históricamente, la escolarización buscó negar la presencia de los medios y de las TIC en los ámbitos institucionales educativos (Huergo \& Fernández, 2000). A pesar de ello, estas problemáticas ingresaban a los espacios educativos: no solo por la presencia de un dispositivo tecnológico disruptivo, en un primer momento, sino también por convertirse en uno de los principales ejes de estructuración de la vida en común. Pero el problema que se presentaba era que los 
dispositivos ingresaban de todas maneras a la escuela. Lo hacían en los bolsillos, en las mochilas, escondidos entre carpetas y cuadernos. Con el PCl, es el Estado el que permite y el que avala el ingreso de las tecnologías a los establecimientos educativos; allí radica un cambio sustancial.

¿Es posible encontrar lazos entre los umbrales que deja el PCl y la comunicación y educación popular? La pregunta nos retrotrae a la escena y a la foto: en esos sentidos se abriga una esperanza. La educación popular, tal como la concibo, se define por el proyecto político que contiene en sus entrañas y por las disputas que establece con los antagonismos presentes en una sociedad en un contexto determinado. Ese proyecto político es contestatario e insumiso frente a las múltiples formas de la opresión (Freire, 1999), e involucra una suma de acciones destinadas a la toma de la palabra por parte de los sectores más postergados.

En ocasiones, distintos colectivos que realizaban acciones de comunicación y educación popular confundieron algunas de sus estrategias de intervención y lograron instalar un imaginario de representaciones donde las TIC eran denostadas por una supuesta pérdida del vínculo humano que subyacían en su apropiación. También se extendió una articulación discursiva que ligaba la educación popular con la ausencia de tecnologías, la carencia de recursos estéticos o la linealidad de las producciones diseñadas y las obtenidas. Aún es factible hallar resonancias de estas perspectivas que tienden a confundir la pobreza de recursos y estrategias a utilizar en propuestas de comunicación y educación popular, con la pobreza económica estructural de los contextos en los cuales intervienen.

Estas creencias extendidas que obturaron, en parte, la presencia de las TIC en procesos de comunicación y educación popular soslayan el vínculo que tuvieron en las experiencias pioneras. Sólo mencionaré algunas, tal vez las más clásicas, para ilustrar esta relación. Luego de la Primera Guerra Mundial, en la campiña francesa, Célestin Freinet utilizó un mimeógrafo para que sus estudiantes confeccionaran un periódico escolar, donde "escribieran para ser leídos». En Colombia, en 1947, el sacerdote Joaquín Salcedo comprende la potencia política del transmisor y funda Radio Sutatenza, pionera en materia de comunicación y educación popular. Algo similar ocurrió en 1953 en Bolivia con las Radios Mineras, y en 1959 en Cuba con Radio Rebelde. Paulo Freire sugería que se proyectaran materiales audiovisuales en los Círculos de Cultura, como 
una herramienta para la postalfabetización y para incrementar la toma de la palabra de hombres y mujeres. Mario Kaplún fue quien hizo conocida la técnica de educación popular llamada cassette-foro, a partir del grabador, y de los talleres de radiograma.

Probablemente, la lista estará plagada de omisiones insensibles de algunas experiencias en comunicación y educación popular (todavía es necesario realizar una cartografía de estas iniciativas, ya bien entrado el siglo xxı). Aun así, es posible encontrar que existió un vínculo entre las TIC que se hallaban disponibles en un contexto determinado, y que diversos agentes y colectivos políticos reconocieron sus potencialidades y buscaron incorporarlas dentro de sus estrategias de intervención político-cultural. Es verdad que en la actualidad del capitalismo posindustrial asistimos a una proliferación de dispositivos, programas informáticos y redes de transmisión de datos impensados en el escenario de las experiencias mencionadas. Lo que quisiera rescatar es la exploración que hicieron aquellos hombres y mujeres de las TIC.

Un dispositivo tecnológico encierra unas posibilidades de enunciación y de utilización de recursos para la exposición de una lectura del mundo. Son finitas, es decir, no hay un número ilimitado de alternativas: sólo es posible expresar ciertas modalidades discursivas de acuerdo con el contexto histórico que nos atraviesa (Foucault, 2010). No se puede hablar de cualquier cosa en cualquier época.

Las TIC nunca fueron neutras ni asépticas. Eso es obvio. Las experiencias aludidas tienen en común un horizonte político que se comprometió con las luchas por la emancipación latinoamericana y por promover instancias de participación y toma de la palabra de los sectores oprimidos. Es el horizonte político que enarbolan hombres y mujeres comprometidos con la comunicación y educación popular el que direcciona las posibilidades de enunciación de un yo discursivo (individual o colectivo) que contiene las TIC. Nunca ocurre de manera inversa: es una falacia sostener esto.

El PCI complejiza algunas de las tradiciones de comunicación y educación popular. Las netbooks tienen la posibilidad de utilizar programas informáticos de código cerrado y otros de código abierto. Jóvenes y docentes pueden optar por diseñar sus estrategias de enseñanza y aprendizaje en cualquiera de los dos. En esa sola posibilidad de elección de usar un tipo de programas se condensa una de las tensiones históricas que atraviesan estructuralmente a América Latina. En palabras de Simón Rodríguez (1990), se trata de la disputa entre lengua y gobierno, que quien busca instalar una lengua también anhela imponer una forma de gobierno que se desprende de la operación 
anterior. Rodríguez esto lo veía en las contiendas por la liberación de España y en los embates de la Real Academia Española por trasladar sus vocaciones imperiales camufladas en un interés por la ortografía. Eso es lo que intentó hacer evidente el maestro de Bolívar con la tecnología de que disponía en su tiempo: la imprenta. Un lenguaje (de código abierto) nuevo puede insuflar horizontes existenciarios no imaginados hasta el momento. De esos horizontes la performatividad se encauza en organización y en elementos de disputa política que persistan en la imitación de las soluciones europeas para los problemas de América. Aquí se vuelve imperativa la todavía vigente proclama: «O inventamos o erramos» (Rodríguez, 1990: 88).

Otra zona de contacto del PCı con las memorias de comunicación y educación popular se aloja en los reconocimientos de las subjetividades en las cuales confiar y creer que son capaces de jugar el juego de la construcción de un mundo más justo. Simón Rodríguez parecía gritar en sus escritos: «DÉNSEME LOS MUCHACHOS POBRES»; mientras que Freire hacía lo mismo con los «desharrapados del mundo». La llegada de las netbooks a muchos hogares se transformó en un acontecimiento inaugural, con momentos de registros fotográficos familiares que pudieran representar la alegría del momento (Ministerio de Educación, 2014). En ello se edificaba una instancia germinal de la construcción de un proyecto político-cultural, en una de sus dimensiones, que apuntara a la instalación de un nuevo orden de significación y de restitución de derechos. El PCI tuvo como destinatarios primordiales a estudiantes y a docentes de todas las escuelas secundarias públicas del país. Con todas las dificultades de implementación que surgieron de una política pública tan vasta, con la desigualdad de los puntos de partida que tienen los/las jóvenes de la Argentina, el PCı pareció asentarse en una de las observaciones agudas de Simón Rodríguez en el siglo XIX para desde allí irradiar su fundamento político hacia el futuro: «Es regular que la Clase Gobernadora tenga Escuelas Privadas - la otra debe conformarse con el destino que la Providencia le da al nacer» (Rodríguez, 1990: 85). El Estado, sí, y la educación pública asumieron los distintos puntos de partida y que la meritocracia es una estrategia retórica liberal que esconde la desigualdad de nuestro continente.

Vuelvo a la escena y a la foto. En las fotos de su beba que esa mamá y ese papá habrán subido - probablemente - a su Facebook, en los posteos a Wikipedia del estudiante acerca de los derechos humanos, subyacen universos significativos que se dispararon a partir de la netbook del PCı. También a esa dimensión de apropiación de la tecnología que se supone desde posiciones biempensantes (¿clasemedieras?) será necesario agregar una dimensión afectiva que se potencia en las prácticas vinculadas con las TIC. 
Ahí es donde, quienes abrevamos en una perspectiva de comunicación y educación popular, deberemos alentar el trabajo político amoroso que reconecte los horizontes políticos de nuestras memorias con las nuevas dimensiones de las afectividades que sustentan las lecturas del mundo de los jóvenes de sectores populares. Sobre todo, porque se vienen tiempos donde habrá que endurecerse sin perder la ternura. Pero tengamos paciencia, porque nuestro tiempo es inconmensurablemente largo. 


\section{Referencias bibliográficas}

Foucault, M. (2010). La arqueología del saber. Buenos Aires, Argentina Siglo XXI.

Freire, P. (1999). Pedagogía del oprimido. México D. F., México: Siglo XXI.

Huergo, J. y Fernández, M. B. (2000). Cultura escolar, cultura mediática / Intersecciones. Bogotá, Colombia: Universidad Pedagógica Nacional.

Ministerio de Educación (2014). Cambios y continuidades en la escuela secundaria: la universidad pública conectando miradas. Estudios evaluativos sobre el Programa Conectar Igualdad. Segunda Etapa. Buenos Aires, Argentina: Ministerio de Educación.

Rodríguez, S. (1990). Sociedades americanas. Caracas, Venezuela: Biblioteca Ayacucho.

\section{Referencia electrónica}

Artiguenave, Darío (2015). Algunos apuntes sobre la formación docente. En Contexto. Recuperado de http://www.diariocontexto.com. ar/2015/09/27/algunos-apuntes-sobre-la-formacion-docente/ 